\title{
Distinguishing primary from secondary mucinous ovarian tumors: an algorithm using the novel marker DPEP1
}

\author{
Takako Okamoto ${ }^{1}$, Noriomi Matsumura ${ }^{1}$, Masaki Mandai ${ }^{1}$, Tomonori Oura ${ }^{2}$, \\ Yukio Yamanishi ${ }^{3}$, Akiko Horiuchi ${ }^{4}$, Junzo Hamanishi ${ }^{1}$, Tsukasa Baba ${ }^{1}$, \\ Masafumi Koshiyama ${ }^{3}$, Tanri Shiozawa ${ }^{4}$ and Ikuo Konishi ${ }^{1}$
}

${ }^{1}$ Department of Gynecology and Obstetrics, Kyoto University Graduate School of Medicine, Kyoto, Japan;

${ }^{2}$ Department of Biostatistics, Kyoto University School of Public Health, Kyoto, Japan; ${ }^{3}$ Departments of

Obstetrics and Gynecology, Otsu Red Cross Hospital, Otsu, Japan and ${ }^{4}$ Department of Obstetrics and

Gynecology, Shinshu University School of Medicine, Matsumoto, Japan

\begin{abstract}
Distinguishing primary mucinous ovarian cancers from ovarian metastases of digestive organ cancers is often challenging. Dipeptidase 1 was selected as the candidate novel marker of colorectal cancer based on an analysis of a gene expression microarray. Immunohistochemical analysis indicated that 13/16 ovarian metastases of colorectal cancers, but only 1/58 primary mucinous ovarian cancers, were dipeptidase 1-positive (threshold; $\geqq 25 \%$ expression, $P<0.0001$ ). Next, five immunohistochemical markers (dipeptidase 1 , estrogen receptor- $\alpha$, cytokeratin 7 , cytokeratin 20 , and caudal type homeobox 2 ) were analyzed in combination. In a hierarchical clustering analysis, the mutually exclusive expression of cytokeratin 7 and dipeptidase 1 specifically identified the ovarian metastases of colorectal cancers $(P<0.0001)$. In a decision tree analysis, cytokeratin 7 , caudal type homeobox 2 , and dipeptidase 1 classified primary mucinous ovarian cancers and ovarian metastases of digestive organ cancers with $90 \%$ accuracy. Finally, the five immunohistochemical markers were combined with six preoperative factors (patient's age, tumor size, laterality, serum CEA, CA19-9, and CA125) and combinations were analyzed. Of the 11 factors, 4 (dipeptidase 1, cytokeratin 7 , caudal type homeobox 2, and tumor size) were used to generate a decision tree to classify primary mucinous ovarian cancers and metastases of digestive organ cancers with $93 \%$ accuracy. In conclusion, we identified a novel immunohistochemical marker, dipeptidase 1, to distinguish primary mucinous ovarian cancers from ovarian metastasis of colorectal cancers. The algorithm using immunohistochemical and clinical factors to distinguish metastases of digestive organ cancers from primary mucinous ovarian cancers will be useful to establish a protocol for the diagnosis of ovarian metastasis.
\end{abstract}

Modern Pathology (2011) 24, 267-276; doi:10.1038/modpathol.2010.204; published online 12 November 2010

Keywords: differential diagnosis; dipeptidase 1(DPEP1); metastasis; mucinous adenocarcinoma; ovary

The ovary is a common site of metastasis, ${ }^{1}$ and in many cases, there is a known history of primary tumors outside the ovary. However, ovarian masses are sometimes found in patients with no known history of malignancy and the primary tumor is not diagnosed until some time later. The

Correspondence: Dr M Mandai, MD, PhD, Department of Gynecology and Obstetrics, Kyoto University Graduate School of Medicine, 54 Shogoin Kawahara-cho, Sakyo-ku, Kyoto, 6068507, Japan.

E-mail: mandai@kuhp.kyoto-u.ac.jp

Received 11 June 2010; revised 7 September 2010; accepted 7

September 2010; published online 12 November 2010 morphology of a metastatic tumor in the ovary can mimic a primary ovarian tumor by a so-called maturation phenomenon. ${ }^{2}$ A majority of the tumors exhibit cystic pattern when they metastasize to the ovary, whereas primary tumor in the original site is not cystic at all. Furthermore, many metastatic mucinous adenocarcinomas, especially from digestive organ cancers, contain a mixture of mucinous epithelium with a range of atypia, resembling primary mucinous carcinomas or borderline tumors. Many metastatic mucinous carcinomas of the ovary have been and continue to be misdiagnosed as primary ovarian mucinous adenocarcinomas. $^{3}$ 
In problematic cases, the diagnosis has been made by integrating clinical, radiological, serological, and pathological features. ${ }^{4}$ Recently, immunohistochemistry, especially cytokeratin 7 (CK7) and cytokeratin 20 (CK20) staining, has been widely used to distinguish primary from secondary ovarian tumors. ${ }^{5,6}$ Although immunohistochemistry has been useful in diagnosis of some cases, current immunohistochemical markers are not sufficient to diagnose all secondary ovarian tumors. ${ }^{7}$ Therefore, further investigation of immunohistochemical markers is required.

In this study, we utilized genome-wide gene expression analysis to identify a novel molecular marker, dipeptidase 1 (DPEP1). We also developed an algorithm to distinguish primary mucinous ovarian tumors from ovarian metastases of digestive organ cancers. This study will be useful to establish a protocol to improve the diagnosis of ovarian metastasis in problematic cases.

\section{Materials and methods}

\section{Tissue Samples}

We collected formalin-fixed and paraffin-embedded tissue sections of primary ovarian mucinous tumors and secondary ovarian carcinomas operated between 1985 and 2008 at Kyoto University Hospital, Shinshu University Hospital, and Otsu Red Cross Hospital (104, 10, and 18 samples, respectively). We received written consent from each patient and approval by the ethics committee of each institute. In this study, only the cases with clinically evident primary sites were used. Tumors were classified as metastatic when they exhibited the same morphologic features as primary site. A total of 122 tumor samples, 86 from primary mucinous ovarian tumors and 36 from metastatic ovarian tumors (Table 1), were used for the following analyses.

\section{Microarray Data Set}

Microarray data (GSE2109) was obtained from the Gene Expression Omnibus (GEO) website (http:// www.ncbi.nlm.nih.gov/geo/). This data set contains 247 epithelial ovarian cancers and 394 colorectal adenocarcinomas arrayed on the Affymetrix U133 plus 2 GeneChip.

\section{Immunohistochemistry}

Immunohistochemical stains were performed using the streptavidin-biotin-peroxidase method. Samples were deparaffinized and antigen retrieval was performed as indicated below. For CK20, estrogen receptor- $\alpha$ (ER), and caudal type homeobox 2 (CDX2) staining, the samples were boiled in $10 \mathrm{mM}$ citrate

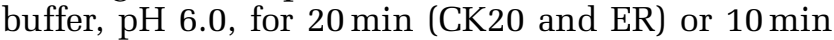
(CDX2). For CK7 staining, the samples were
Table 1 Number of cases used for the analyses

Number

of cases

\begin{tabular}{|c|c|c|}
\hline Primary ovarian tumors & 86 & \\
\hline Mucinous adenoma & 28 & \\
\hline Mucinous borderline tumor & 28 & \\
\hline Intestinal type & 15 & \\
\hline Endocervical type & 13 & \\
\hline Mucinous adenocarcinoma (stage I) & 24 & \\
\hline Mucinous adenocarcinoma (stage III) & 6 & \\
\hline Secondary ovarian tumors & 36 & \\
\hline Colorectal cancer & 16 & \\
\hline Appendix cancer & 2 & \\
\hline Small intestine cancer & 1 & Digestive organ \\
\hline Pancreatic cancer & 2 & cancer other than \\
\hline Bile duct cancer & 3 & colorectal cancer \\
\hline Gastric cancer & 8 & \\
\hline Breast cancer & 4 & \\
\hline
\end{tabular}

incubated for $20 \mathrm{~min}$ at $37^{\circ} \mathrm{C}$ with pepsin (Dako, Glostrup, Denmark). For DPEP1 staining, the samples were heated at $98{ }^{\circ} \mathrm{C}$ for $15 \mathrm{~min}$ in Dako solution. To block endogenous peroxidase activity, $100 \%$ methanol containing $0.3 \% \mathrm{H}_{2} \mathrm{O}_{2}$ was added for 15 min. Nonspecific binding of IgG was blocked using normal rabbit (for CK20, CK7, ER, and CDX2) or goat serum (for DPEP1) (Nichirei, Tokyo, Japan). The sections were incubated overnight at $4{ }^{\circ} \mathrm{C}$ with the following antibodies: CK20; clone Ks20.8 (Novocastra, New Castle, UK; ready-to-use), CK7; clone OV-TL 12/30 (DAKO; ready-to-use), ER; clone 6F11 (DS Pharma Biomedical, Japan, 1:40), CDX2; clone CDX2-88 (Biogenex, San Ramon, CA, USA; ready-touse), and DPEP1; HPA012783 (Sigma-Aldrich, St Louis, MO, USA; 1:50). Next, sections were incubated with biotinylated anti-mouse (for CK20, CK7, ER, and CDX2) or anti-rabbit (DPEP1) secondary antibodies (Nichirei). Subsequently, sections were incubated with streptavidin-peroxidase complex solution for $30 \mathrm{~min}$. Signals were generated by incubation with 3,3'-diaminobenzidine. Finally, the sections were counterstained with hematoxylin.

\section{Hierarchical Clustering}

Hierarchical clustering was performed using Cluster 3.0 software (http://rana.lbl.gov/EisenSoftware.htm). The green-black-red heatmap was drawn by Java TreeView 1.1.2 (http://jtreeview.sourceforge.net/). The blue-yellow-red heatmap was drawn by R 2.8.1 (http://www.R-project.org.).

\section{Decision Tree Analyses}

The WEKA data mining software was downloaded from http://www.cs.waikato.ac.nz/ ml/index.html. For the decision tree analyses, the WEKA J48 tree classifier was used. 


\section{Statistical Analysis}

All the statistical analyses were performed using GraphPad Prism 4.0b (GraphPad Software, La Jolla, CA, USA). ROC curves were generated using Microsoft Excel 2007 (Microsoft, Tokyo, Japan).

\section{Results}

Investigation of a Novel Immunohistochemical Marker to Distinguish Primary from Secondary Ovarian Cancer

We analyzed microarray data set GSE2109, which contains 247 epithelial ovarian cancers and 394 colorectal cancers. The fold difference of average expression between the ovarian and the colorectal cancers were determined for each of the U133 plus 2 gene probes $(n=54613)$. A threshold of tenfold difference identified 40 probes (28 genes) upregulated in ovarian cancers and 114 probes (85 genes) upregulated in colorectal cancers (Supplementary Table 1). Several of these genes encode known immunohistochemical markers used to distinguish primary from secondary ovarian cancers, such as CK7, ER, CK20, and CDX $2^{8-10}$ (Figure 2). FOLR1, ${ }^{11}$ MUC16, ${ }^{12}$ PAX8, ${ }^{13}$ WT1, ${ }^{14}$ CEACAM-1, 5, 6, 7, ${ }^{12}$ $M E P 1 A,{ }^{15}$ and $M U C 2^{16}$ were also identified (Supplementary Table 1). Therefore, this gene list was expected to include novel marker genes that could be used to discriminate primary from secondary ovarian cancers. DPEP1, which is reported to be expressed in colorectal cancer, ${ }^{17,18}$ was among the genes identified (Figure 2).

We next determined if DPEP1 protein expression could be used to distinguish primary mucinous ovarian carcinomas (including borderline tumors) from ovarian metastases of colorectal cancers. DPEP1 expression was localized to the brush border (Figure 1b) and was observed in varying percentages of tumor cells in different samples. We quantified the percentage of positive cells in each sample. The majority of metastatic colorectal cancer samples contained $>25 \%$ DPEP-1-positive cells (13/16), whereas nearly all primary mucinous ovarian carcinomas samples contained $<25 \%$ DPEP-1-positive cells $(1 / 58 ; P<0.0001$, Fisher's exact test; Figure 1c). These results indicate that DPEP1 may be useful as an immunohistochemical marker to distinguish primary from secondary mucinous ovarian carcinoma.

\section{Combined Analysis Using Multiple Immunohistochemical Markers}

Expression levels of CK7, ER, CK20, and CDX2 were analyzed by immunohistochemistry. ER and CDX2 were found in the nucleus, whereas CK7 and CK20 were found in the cytoplasm (Figure 2a and b). Most samples had heterogeneous protein expression. In each sample, we quantified the percentage of tumor cells positive for each protein. In preliminary analyses, the best threshold of the percentage of expression of positive cells to distinguish primary from secondary ovarian cancers varied from protein to protein (data not shown). Therefore, we utilized the actual percent expression rather than determining arbitrary thresholds in the subsequent analyses. We performed a hierarchical clustering analysis to examine the associations between the five molecules. Notably, ovarian metastases of colorectal cancers were distinguished from other tumors by the mutually exclusive expression of CK7 and DPEP1 $(P<0.0001$, Fisher's exact test; Figure 2c). Ovarian metastases of non-colorectal digestive organ cancers exhibited modest expression of CK7, CK20, and CK20. Ovarian metastases of breast cancer were distinguished from other metastases by abundant ER and CK7 expression and lack of CK20, CDX2, and DPEP1 expression. DPEP1 was negatively correlated with CK7 $(r=-0.66, P<0.0001$, Pearson's correlation) and positively correlated with CK20 $(r=0.26$, $P=0.0037)$ and CDX2 $(r=0.49, P<0.0001)$.

Next, we generated dot plot graphs for the percent expression of each protein in each tumor type (Figure 2d). The percent positivity was different among groups (ER, $P=0.0017$; others, $P<0.0001$, Kruskal-Wallis test). The ovarian metastases of colorectal cancers showed the least prevalent expression of CK7 and the most abundant expression of CK20, CDX2, and DPEP1. The ovarian metastases of non-colorectal digestive organ cancers had expression similar to but less striking than the metastases of colorectal cancers. A comparison of primary mucinous ovarian carcinomas (including borderline tumors) and metastases of digestive organ cancers (colorectal plus non colorectal) indicated that CK7 expression was more abundant in primary mucinous ovarian carcinomas, whereas CK20, CDX2, and DPEP1 expression was more abundant in metastases of digestive organ cancers (Figure $3 \mathrm{~b}$; CK7, CDX2, and DPEP1, $P<0.0001 ; \quad$ CK20, $P=0.0003$, Mann-Whitney $U$ test). ER expression tended to be more abundant in primary mucinous ovarian carcinomas than the metastases of digestive organ cancers, but the difference was not statistically significant $(P=0.11)$.

Given the distinct immunohistochemical patterns in ovarian metastases of colorectal cancers and the modest but the similar pattern in metastases of noncolorectal digestive organ cancers, a combination of the immunohistochemical markers was expected to distinguish primary mucinous ovarian carcinomas (including borderline tumors) from metastases of digestive organ cancer. The Weka J48 software determines a decision tree that best discriminates between different groups using the minimum number of factors. Among the five molecules, three (CDX2, CK7, and DPEP1) were selected to draw a decision tree (Figure 2e) with the net accuracy of $90 \%$ (81/90). In all, 97\% (56/58) of the primary mucinous ovarian carcinomas and $100 \%(16 / 16)$ of the ovarian metastases of colorectal cancers were 


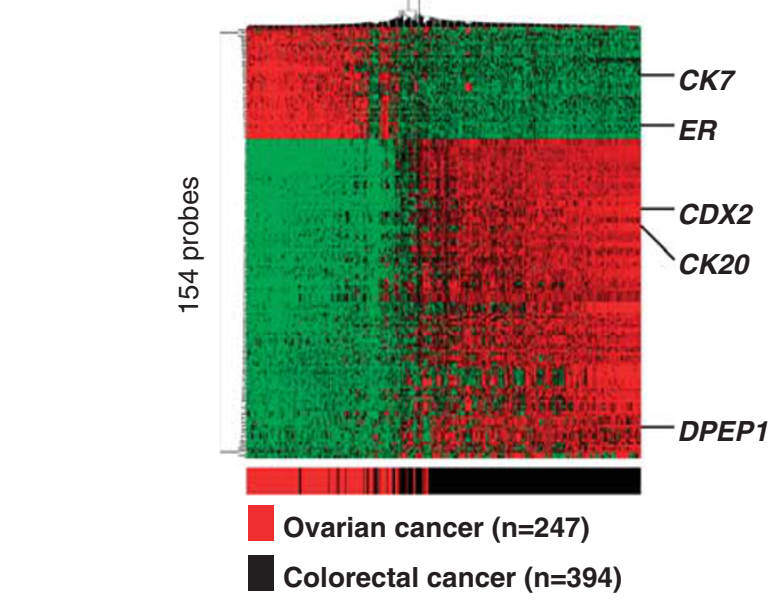

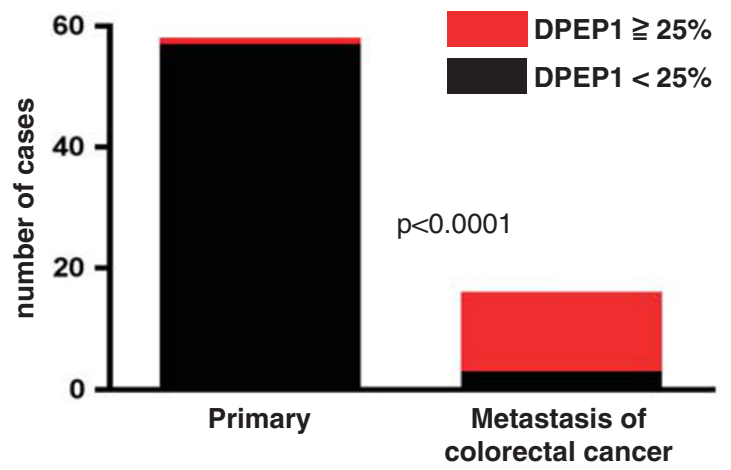

b

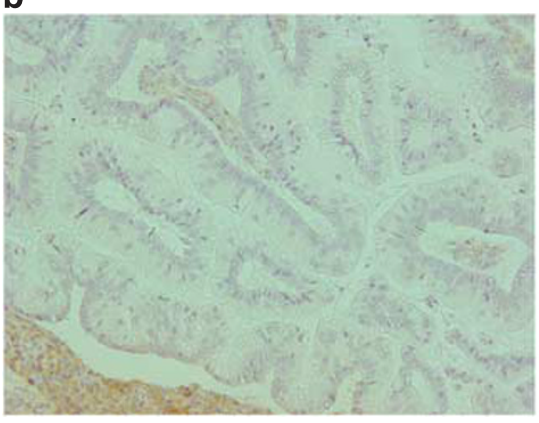

primary $(0 \%)$

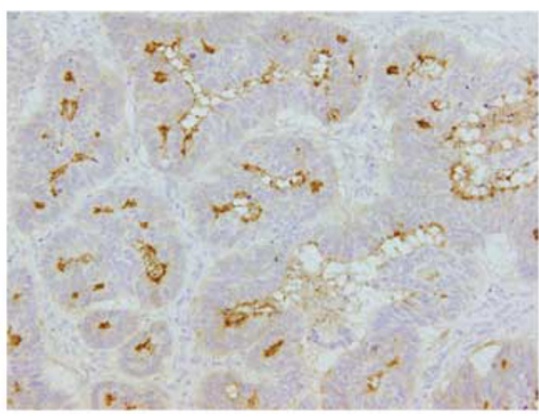

Metastasis of colorectal cancer

$(100 \%)$

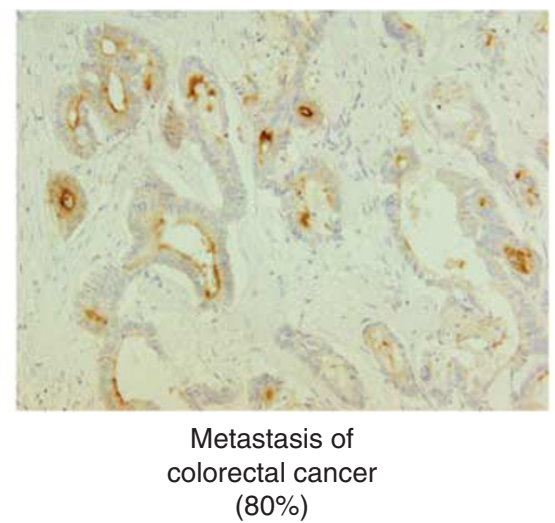

$(80 \%)$

Figure 1 Identification of a novel immunohistochemical marker DPEP1 to distinguish primary mucinous ovarian carcinomas from ovarian metastases of colorectal cancers. (a) Identification of genes differentially expressed in ovarian cancer and colorectal cancer. A total of 154 probes were selected using a cutoff of tenfold difference in expression. Gene expressions are $\log _{2}$ transformed, centered, and normalized, and then the genes and tumor samples are ordered by an average-linkage hierarchical clustering. (b) Representative images of DPEP1 immunostaining in ovarian metastases of colorectal cancers and primary mucinous ovarian carcinomas. The percentage of positive tumor cells in the sample is indicated. (c) Number of samples with $\geqq 25 \%$ or $<25 \%$ DPEP1-positive tumor cells in primary mucinous ovarian carcinomas and ovarian metastases of colorectal cancers cases is shown.

correctly discriminated. Among the ovarian metastases of non-colorectal digestive organ cancers, the accuracy was $56 \%(9 / 16)$.

\section{Combined Analysis Using Immunohistochemical Markers and Preoperative Clinical Information}

The preoperative clinical information was added to improve the accuracy with which primary mucinous ovarian carcinomas (including borderline tumors) were distinguished from ovarian metastases of digestive organ cancers. The patient's age at the date of surgery was significantly different $(P=0.032$, Kruskal-Wallis test) between the groups (Figure 3a). The patient's age of the metastatic digestive organ cancers was significantly higher than that of primary mucinous ovarian carcinomas (median age: 57 vs 46.5 years old, $P=0.0027$, Mann-Whitney $U$ test). The longest diameters of the tumors were significantly different between the groups
$(P=0.0003)$. Tumor sizes of ovarian metastases of digestive organ cancers were significantly smaller than that of primary mucinous ovarian carcinomas (Figure 3a; median size: 10 vs $15 \mathrm{~cm}, P<0.0001$ ). The percentage of bilateral tumors was significantly higher in ovarian metastases of digestive organ cancers than in primary mucinous ovarian carcinomas (Figure 3a; 35 vs 14\%, $P=0.020$ ). Seidman et $a l^{3}$ previously reported that laterality and tumor size correctly classified $90 \%$ of the primary and secondary ovarian cancers. In our data set, Seidman's rule identified primary mucinous ovarian carcinomas and ovarian metastases of digestive organ cancers with the accuracy of $71 \%$ (62/87).

We next examined the preoperative serum concentration of CEA, CA19-9, and CA125. Each was different among the groups (Figure 3b; CEA, $P<0.0001$; CA19-9, $P=0.0010 ; \quad$ and CA125, $P=0.0002$, Kruskal-Wallis test). Comparison of ovarian metastases of digestive organ cancers and 
primary mucinous ovarian carcinomas (including borderline tumors) indicated that the former showed a higher serum CEA values than the latter (median
6.5 vs $1.3 \mathrm{ng} / \mathrm{ml}, P<0.0001)$. CA19-9 and CA125 values were not significantly different between samples. a

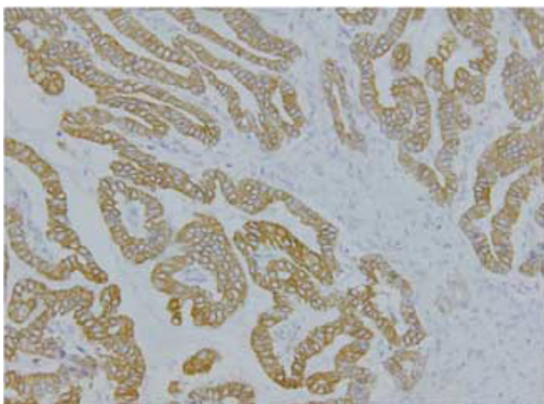

primary $(100 \%)$

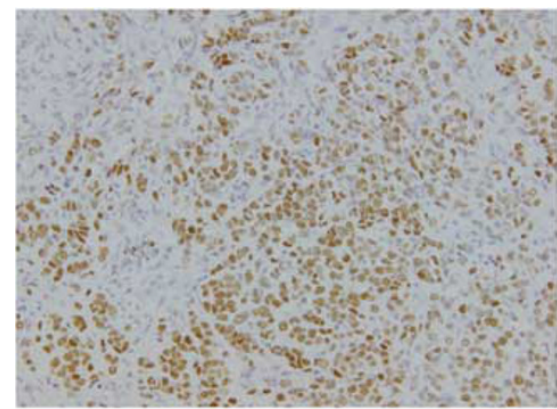

Metastasis of breast cancer (90\%)

b

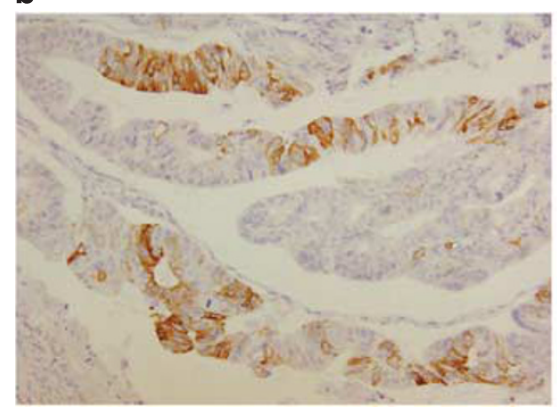

primary (35\%)

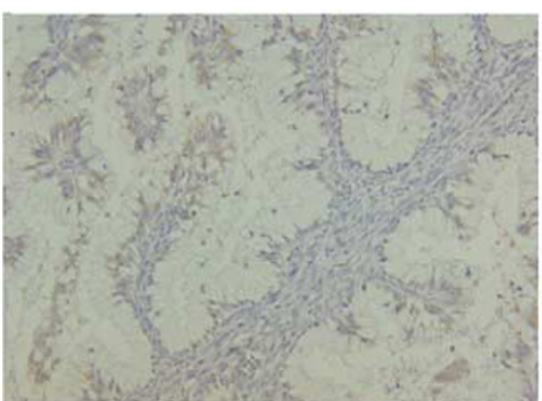

primary $(0 \%)$

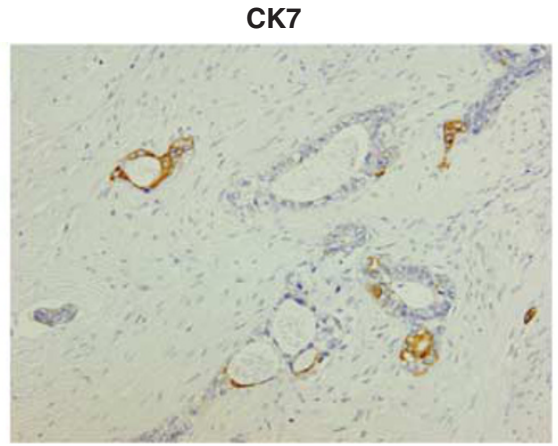

Metastasis of colorectal cancer $(20 \%)$

ER

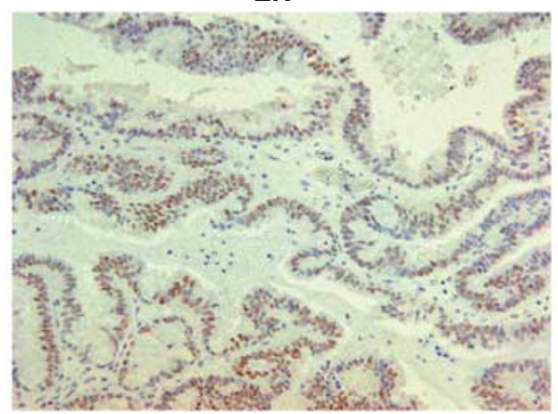

primary $(70 \%)$

CK20

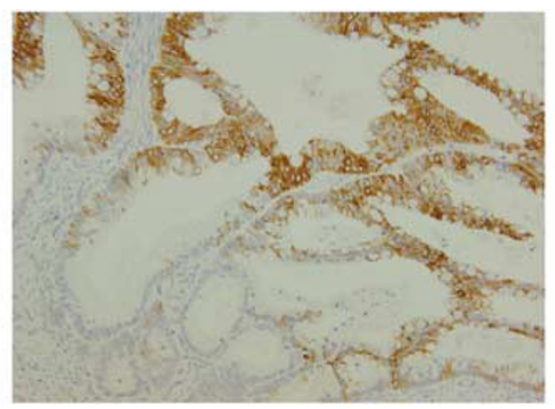

primary $(60 \%)$

CDX2

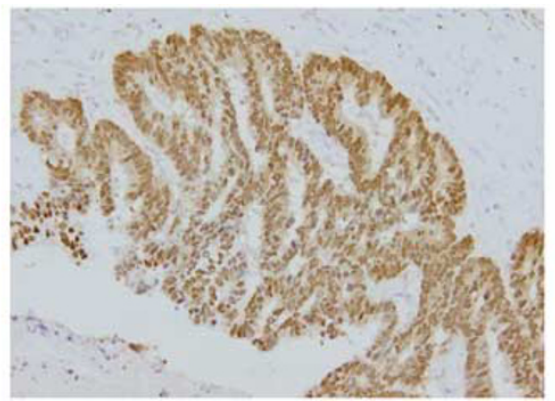

Metastasis of colorectal cancer (100\%)

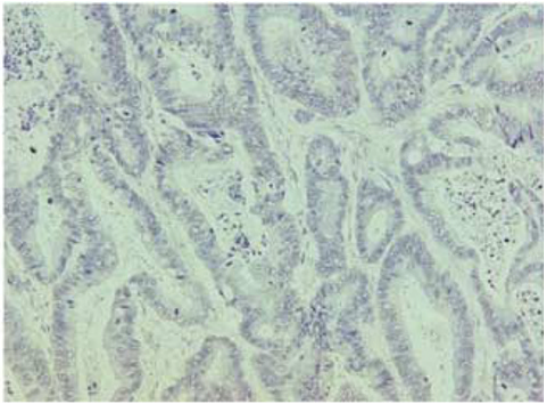

Metastasis of

colorectal cancer $(0 \%)$

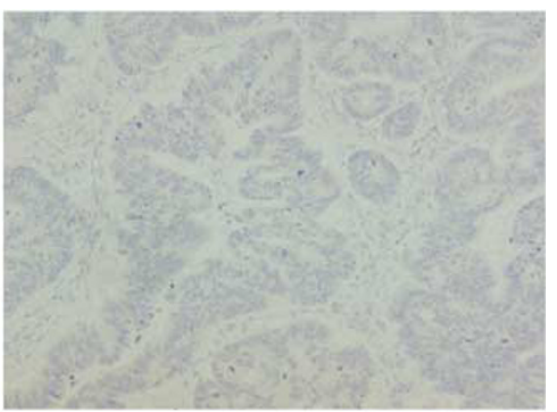

Metastasis of colorectal cancer $(0 \%)$

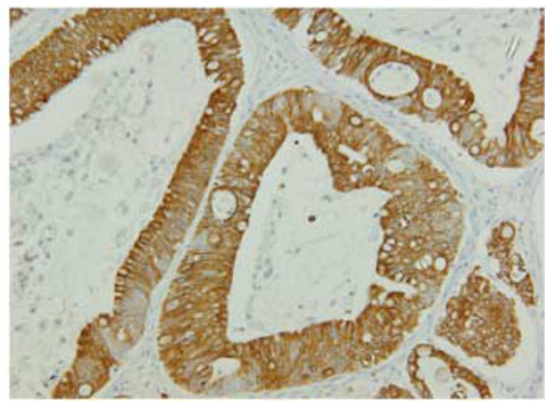

Metastasis of colorectal cancer $(90 \%)$

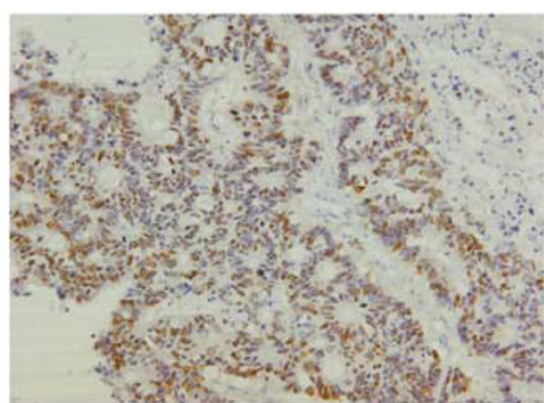

Metastasis of colorectal cancer $(70 \%)$

Figure 2 For caption see next page. 

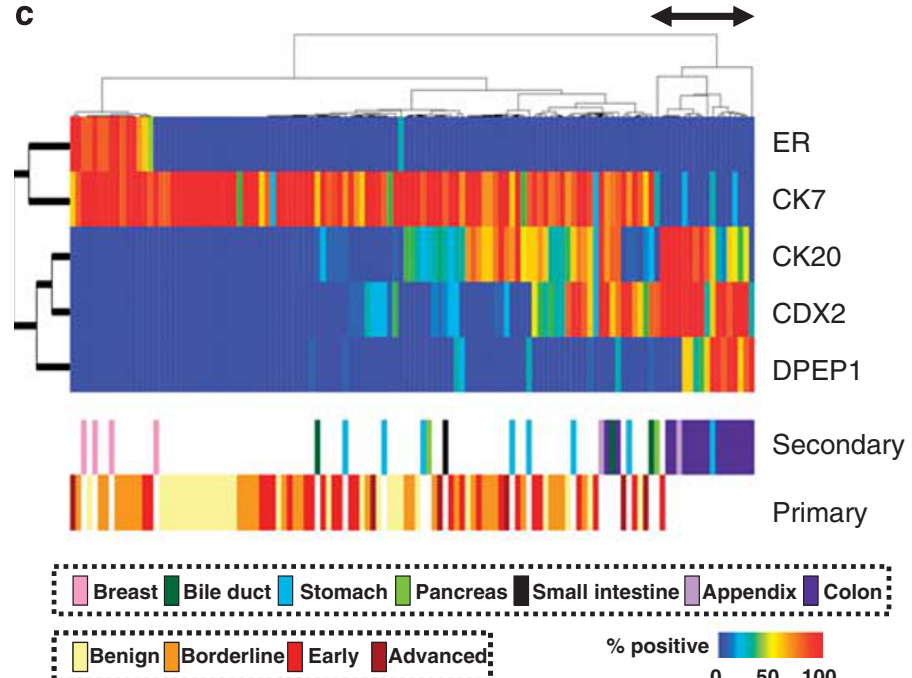

e

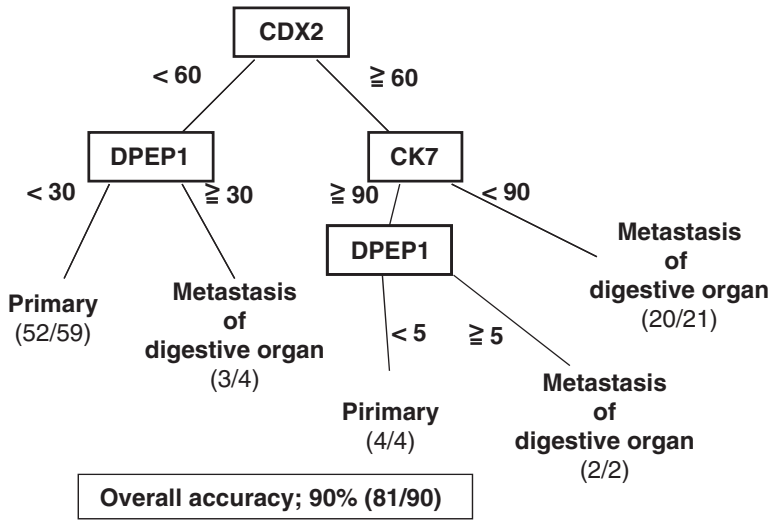

d
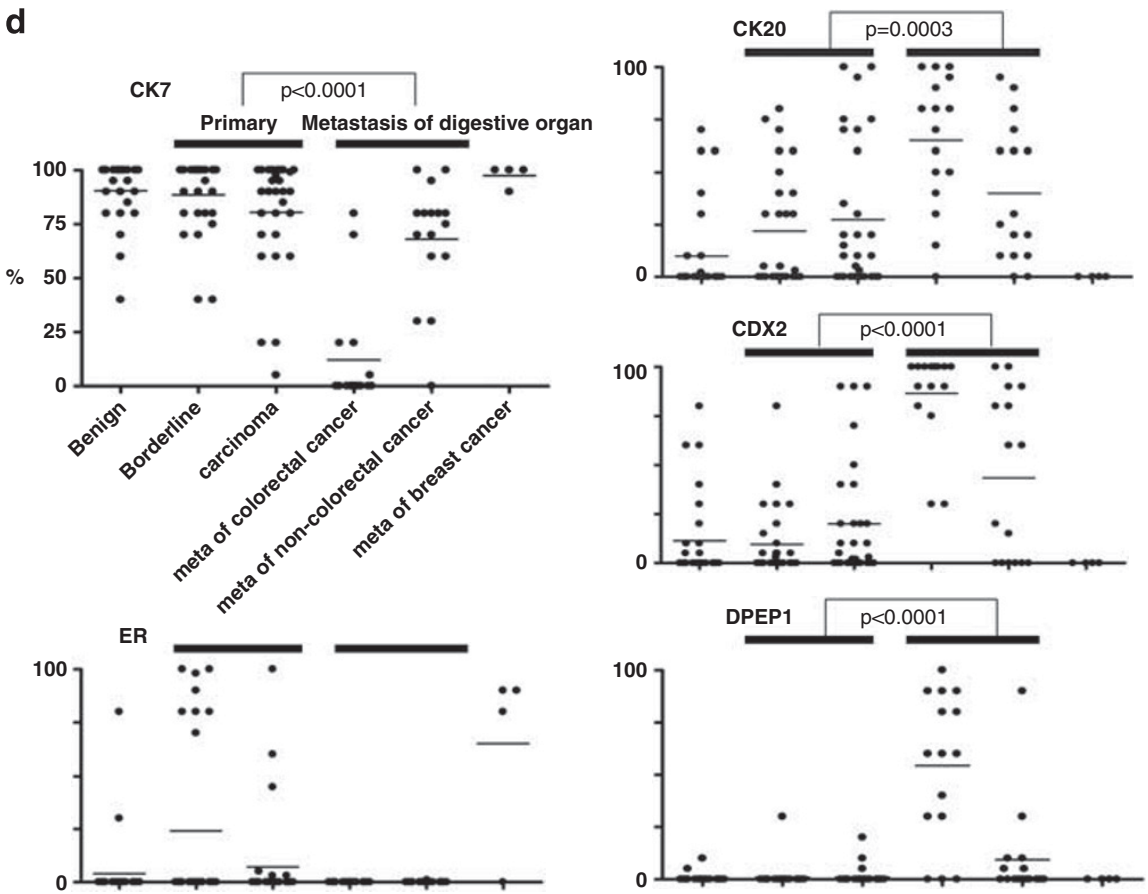

Figure 2 Analyses using multiple immunohistochemical markers. (a) Representative images of CK7, ER, CK20, and CDX2 immunostaining. (b) An average-linkage hierarchical clustering analysis by the percentage immunohistochemical positivity of the five molecules in the individual samples. The percentage positivity is shown by the blue-yellow-red heatmap. The double-headed arrow indicates a tumor cluster determined by scarce CK7 and abundant DPEP1, enriching ovarian metastases of colorectal cancers $(P<0.0001$, Fisher's exact test). Secondary; secondary ovarian tumors. The primary sites are indicated by the color bar. Primary; primary mucinous ovarian tumors. Benign tumor, borderline tumor, early-stage (stage I) carcinoma, and advanced-stage (stage III) carcinoma are indicated by the color bar. (c) The percentage positivity of the five molecules in the individual samples is shown on dot graphs. Ovarian metastasis of adenocarcinomas originating in bile duct, stomach, pancreas, small intestine, and appendix are designated as 'ovarian metastases of noncolorectal digestive organ cancers'. (d) Decision tree that best distinguishes primary mucinous ovarian carcinomas from the metastases of digestive organ cancers. Among the five molecules, CDX2, CK7, and DPEP1 were selected to draw the decision tree. 'Primary mucinous ovarian carcinomas (52/59)' indicates that 52 samples were primary mucinous ovarian carcinomas among the 59 samples classified. The numbers on the connecting lines indicate percent positivity. (e) Decision tree using immunohistochemical data for five proteins, among which three factors (CDX2, CK7 and DPEP) were selected to draw the decision trees.

Finally, we employed all factors analyzed in this study (immunohistochemical expression of five protein markers, three clinical features (Figure 3a), and three serum biomarkers (Figure $3 \mathrm{~b}$ )) to draw a decision tree. Among the 11 factors, 4 (CDX2, CK7,
DPEP1, and tumor size) were used to draw a tree that best discriminates between primary mucinous ovarian carcinomas and ovarian metastases of digestive organ cancers with the net accuracy of 93\% (84/90; Figure 3c). 


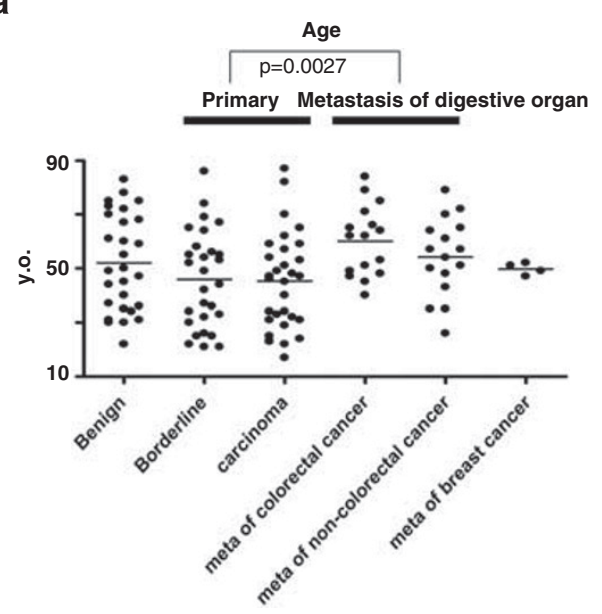

b

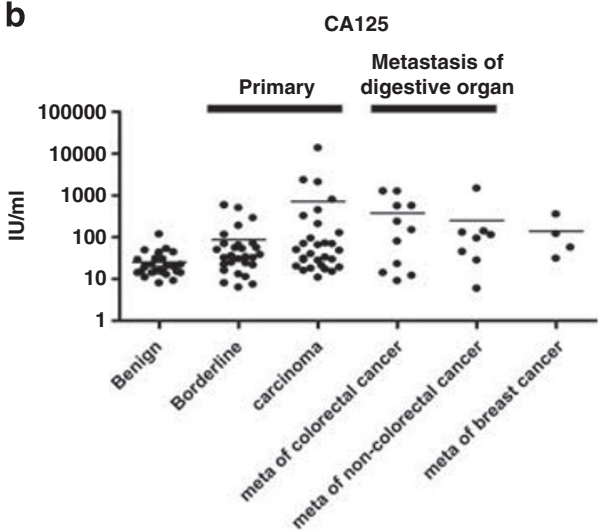

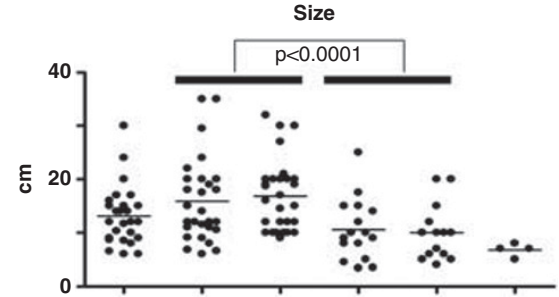
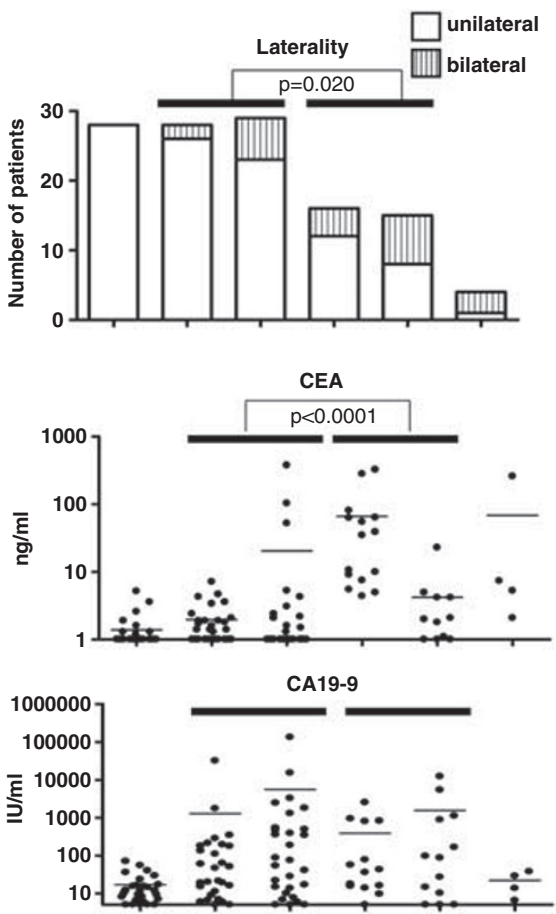

C

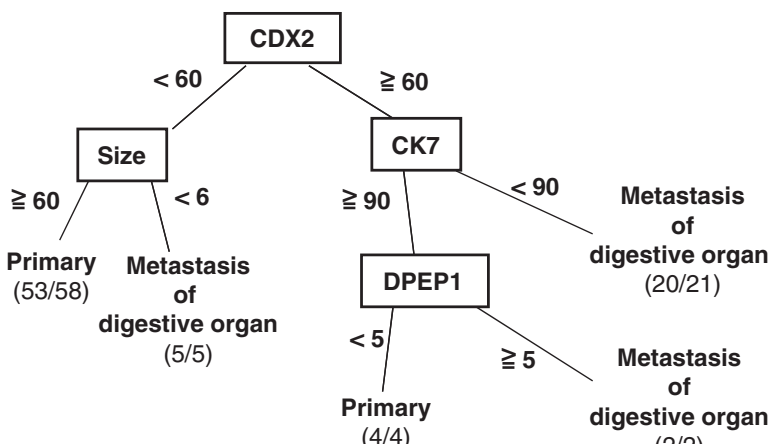

$(2 / 2)$

Overall accuracy; $93 \%(84 / 90)$

Figure 3 Analysis using preoperative clinical information and immunohistochemical markers. (a) Patient's age at the operation, tumor size, and tumor laterality are represented on dot plots. (b) Preoperative serum concentrations of CA125, CEA, and CA19-9 are represented on dot plots. (c) Decision tree using immunohistochemical data for five proteins and six preoperative factors. Among the 11 factors, 4 (CDX2, CK7, DPEP1, and tumor size) were selected to draw the decision tree.

\section{Discussion}

The distinction of primary mucinous ovarian cancers from ovarian metastasis of digestive organ cancers is often difficult. Features common among primary ovarian mucinous neoplasms include unilaterality, size $>150 \mathrm{~mm}$, smooth capsule, and lack of extraovarian spread. In addition, primary ovarian mucinous carcinomas are often accompanied by borderline mucinous tumors. Confluent glandular or expansile invasion is observed in many cases and destructive stromal invasion may also be detected. On the other hand, features common among metastatic mucinous adenocarcinomas of the ovary include bilaterality, small size, surface involvement, nodular involvement, and destructive 
stromal invasion. ${ }^{3,4,19,20}$ Unfortunately, in many cases, the diagnosis is not straightforward because many morphological features are shared by primary and secondary ovarian tumors. ${ }^{7}$ The aim of this research was to develop a protocol to more accurately distinguish primary mucinous ovarian cancer from ovarian metastasis of digestive organ cancer, including colorectal and non-colorectal cancer.

We first sought a novel molecular marker of metastatic ovarian cancer using microarray analysis. A list of genes differentially expressed in epithelial ovarian cancers and colorectal cancers, one of the most common metastatic tumors to involve the ovary, ${ }^{7}$ contained several genes encoding molecular markers known to distinguish primary from secondary ovarian neoplasms (Figure 1a, Supplementary Table 1). Thus, we expected that the list of differentially expressed genes might include novel immunohistochemical markers. Among them, we selected and investigated DPEP1 as a candidate marker because specific antibody was commercially available and its expression pattern in colorectal cancer is known. ${ }^{17,18}$ DPEP1 is a zinc-dependent metallopeptidase that hydrolyzes a variety of dipeptides and is involved in glutathione metabolism. ${ }^{21}$ As expected, positive DPEP1 expression was strongly associated with ovarian metastases of colorectal cancer (Figure 1c). Particularly, its high specificity $(98 \%, 57 / 58$, Figure 1c) to ovarian metastases of colorectal cancer suggests its clinical usefulness as the immunohistochemical marker.

Immunohistochemical expression of CK7, CK20, ER, and CDX2 has been used to distinguish primary from secondary ovarian cancers. ${ }^{8,9,22}$ To evaluate the accuracy that the positivity of each marker predicts primary mucinous ovarian cancer vs ovarian metastasis of digestive organ cancer, we calculated the cutoff value by ROC curve. Each cutoff value of CK7, CK20, CDX2, and DPEP1 classified our cases in the accuracy of $76,64,83$, and $81 \%$, respectively (Supplementary Figure 1). As CK7 and CK20 stainings are most commonly used for the identification of primary and metastatic tumors, we also calculated the accuracy by the combination of CK7/ CK20 staining, which classified primary mucinous ovarian carcinomas and ovarian metastases of digestive organ cancers with only $82 \%$ accuracy in our cases (Supplementary Figure 2). In order to compare DPEP1 with the commonly used markers and to develop an algorithm using multiple markers, we conducted a combined analysis. Hierarchical clustering has commonly been used to analyze DNA microarray data. More recently, studies of protein expression, including ours, have taken advantage of this method. ${ }^{23,24}$ The hierarchical clustering detected the mutually exclusive pattern of CK7 and DPEP1 (Figure 2c). Therefore, a combination of CK7 and DPEP1 staining is expected to distinguish ovarian metastases of colorectal cancers from primary mucinous ovarian carcinomas very accurately. Contrary to the case of colorectal cancers, it is known that CK7, CK20, and CDX2 are not useful as markers to distinguish primary mucinous ovarian carcinomas from ovarian metastases of non-colorectal digestive organ cancers. ${ }^{8,22}$ However, few reports have tested their utility in combination. Therefore, we conducted an analysis by combining multiple immunohistochemical markers. The Weka J48 software, which determines a decision tree that best discriminates between different groups using the minimum number of factors, selected CDX2, CK7, and DPEP1 to draw a decision tree (Figure 2e) with the net accuracy of $90 \%$ (81/90). Using this method, 97\% (56/58) of the primary mucinous ovarian carcinomas and 100\% (16/16) of the ovarian metastases of colorectal cancers were correctly discriminated. Among the ovarian metastases of non-colorectal digestive organ cancers, the accuracy was 56\% (9/16). The fact that DPEP1 expression was included in the decision tree (Figure 2e) indicates that DPEP1 expression provides unique information that was useful to improve prediction.

Recently, clinical information has been re-evaluated to distinguish primary mucinous ovarian carcinoma from ovarian metastasis of digestive organ cancers. Seidman et $a l^{3}$ classified primary and secondary ovarian neoplasms with 90\% accuracy based on tumor laterality and size. Although smaller, bilateral tumors were significantly associated with ovarian metastasis of digestive organ cancers in our data set (Figure 3a), the algorithm of Seidman et $a l^{3}$ classified primary and secondary tumors with an accuracy of only $71 \%$ in our cases. The modified size criteria of Yemelyanova et al $\mathrm{I}^{25}$ classified our cases with only $71 \%$ accuracy. In addition to tumor size and laterality, increased age (Figure 3a) and preoperative serum CEA (Figure 3b) were significantly associated with ovarian metastasis of digestive organ cancers, consistent with a previous report. ${ }^{26}$ To develop a decision tree that more accurately distinguishes primary mucinous ovarian carcinomas from ovarian metastasis of digestive organ cancers, we next attempted to combine all the preoperative clinical information and immunohistochemical data. Of the six preoperative factors (Figure 3a and b), only 'tumor size' contributed to the decision tree (Figure 3c), indicating that immunohistochemical markers are necessary in problematic cases. Notably, DPEP1 was included in the decision tree, again verifying the importance of DPEP1 as the immunohistochemical marker. After all criteria were considered, CDX2, CK7, DPEP1, and tumor size were used to draw a tree that best discriminates primary mucinous ovarian carcinomas from ovarian metastasis of digestive organ cancers with a net accuracy of $93 \%$ (84/90; Figure 3c). We also assessed the accuracy of the Weka J48 tree model by a cross-validation. The accuracy was $83 \%$ when all the 11 factors (5 immunohistochemical and 6 clinical factors) were used. When the five immunohistochemical markers were used, the accuracy was $81 \%$. When just CK7 
and CK20 were used, the accuracy was only $70 \%$. Then, when Seidman's factors (tumor size and laterality) were used, the accuracy was only $67 \%$. Therefore, the cross-validation analysis of the tree model again indicated the accuracy of the prediction by using multiple factors.

In conclusion, using gene expression microarray analysis, we found a novel immunohistochemical marker, DPEP1, which specifically detects metastatic colorectal cancer samples. We also developed an algorithm to distinguish primary mucinous ovarian carcinomas from ovarian metastasis of digestive organ cancers using clinical information and immunohistochemical markers, including DPEP1. This study will be useful to improve the problematic diagnosis of mucinous ovarian cancers and ovarian metastasis from digestive organ cancers. Unfortunately, the accuracy with which primary mucinous ovarian carcinomas were distinguished from ovarian metastasis of digestive organ cancers was not $100 \%$, even when all the factors were used. Especially, distinction of primary mucinous ovarian carcinomas from ovarian metastases of noncolorectal digestive organ cancers is clinically important. For this purpose, it is necessary to identify specific immunohistochemical markers distinguishing them. Gene expression microarray analysis, like that we used to identify DPEP1, should be useful to identify such markers.

\section{Disclosure/conflict of interest}

The authors declare no conflict of interest.

\section{References}

1 Young RH, Scully RE. Metastatic tumors in the ovary: a problem-oriented approach and review of the recent literature. Semin Diagn Pathol 1991;8:250-276.

2 Young RH. From krukenberg to today: the ever present problems posed by metastatic tumors in the ovary: part I. Historical perspective, general principles, mucinous tumors including the krukenberg tumor. Adv Anat Pathol 2006;13:205-227.

3 Seidman JD, Kurman RJ, Ronnett BM. Primary and metastatic mucinous adenocarcinomas in the ovaries: incidence in routine practice with a new approach to improve intraoperative diagnosis. Am J Surg Pathol 2003;27:985-993.

4 Lee KR, Young RH. The distinction between primary and metastatic mucinous carcinomas of the ovary: gross and histologic findings in 50 cases. Am J Surg Pathol 2003;27:281-292.

5 McCluggage WG. Recent advances in immunohistochemistry in gynaecological pathology. Histopathology 2002;40:309-326.

6 McCluggage WG. Recent advances in immunohistochemistry in the diagnosis of ovarian neoplasms. J Clin Pathol 2000;53:327-334.

7 McCluggage WG, Wilkinson N. Metastatic neoplasms involving the ovary: a review with an emphasis on morphological and immunohistochemical features. Histopathology 2005;47:231-247.

8 Vang R, Gown AM, Barry TS, et al. Cytokeratins 7 and 20 in primary and secondary mucinous tumors of the ovary: analysis of coordinate immunohistochemical expression profiles and staining distribution in 179 cases. Am J Surg Pathol 2006;30:1130-1139.

9 Vang R, Gown AM, Barry TS, et al. Immunohistochemistry for estrogen and progesterone receptors in the distinction of primary and metastatic mucinous tumors in the ovary: an analysis of 124 cases. Mod Pathol 2006;19:97-105.

10 Werling RW, Yaziji H, Bacchi CE, et al. CDX2, a highly sensitive and specific marker of adenocarcinomas of intestinal origin: an immunohistochemical survey of 476 primary and metastatic carcinomas. Am J Surg Pathol 2003;27:303-310.

11 Yuan Y, Nymoen DA, Dong HP, et al. Expression of the folate receptor genes FOLR1 and FOLR3 differentiates ovarian carcinoma from breast carcinoma and malignant mesothelioma in serous effusions. Hum Pathol 2009;40:1453-1460.

12 Lagendijk JH, Mullink H, Van Diest PJ, et al. Tracing the origin of adenocarcinomas with unknown primary using immunohistochemistry: differential diagnosis between colonic and ovarian carcinomas as primary sites. Hum Pathol 1998;29:491-497.

13 Nonaka D, Chiriboga L, Soslow RA. Expression of pax8 as a useful marker in distinguishing ovarian carcinomas from mammary carcinomas. Am J Surg Pathol 2008;32:1566-1571.

14 Moritani S, Ichihara S, Hasegawa M, et al. Serous papillary adenocarcinoma of the female genital organs and invasive micropapillary carcinoma of the breast. Are WT1, CA125, and GCDFP-15 useful in differential diagnosis? Hum Pathol 2008;39:666-671.

15 Heinzelmann-Schwarz VA, Scolyer RA, Scurry JP, et al. Low meprin alpha expression differentiates primary ovarian mucinous carcinoma from gastrointestinal cancers that commonly metastasise to the ovaries. J Clin Pathol 2007;60:622-626.

16 Lau SK, Weiss LM, Chu PG. Differential expression of MUC1, MUC2, and MUC5AC in carcinomas of various sites: an immunohistochemical study. Am J Clin Pathol 2004;122:61-69.

17 Yajima S, Ishii M, Matsushita $\mathrm{H}$, et al. Expression profiling of fecal colonocytes for RNA-based screening of colorectal cancer. Int J Oncol 2007;31:1029-1037.

18 McIver CM, Lloyd JM, Hewett PJ, et al. Dipeptidase 1: a candidate tumor-specific molecular marker in colorectal carcinoma. Cancer Lett 2004;209:67-74.

19 Riopel MA, Ronnett BM, Kurman RJ. Evaluation of diagnostic criteria and behavior of ovarian intestinaltype mucinous tumors: atypical proliferative (borderline) tumors and intraepithelial, microinvasive, invasive, and metastatic carcinomas. Am J Surg Pathol 1999;23:617-635.

20 Ronnett BM, Kurman RJ, Shmookler BM, et al. The morphologic spectrum of ovarian metastases of appendiceal adenocarcinomas: a clinicopathologic and immunohistochemical analysis of tumors often misinterpreted as primary ovarian tumors or metastatic tumors from other gastrointestinal sites. Am J Surg Pathol 1997;21:1144-1155.

21 Kozak EM, Tate SS. Glutathione-degrading enzymes of microvillus membranes. J Biol Chem 1982;257: 6322-6327. 
22 Vang R, Gown AM, Wu LS, et al. Immunohistochemical expression of CDX2 in primary ovarian mucinous tumors and metastatic mucinous carcinomas involving the ovary: comparison with CK20 and correlation with coordinate expression of CK7. Mod Pathol 2006;19:1421-1428.

23 Liu M, Matsumura N, Mandai M, et al. Classification using hierarchical clustering of tumor-infiltrating immune cells identifies poor prognostic ovarian cancers with high levels of COX expression. Mod Pathol 2009;22:373-384.

24 Prasad ML, Barbacioru CC, Rawal YB, et al. Hierarchical cluster analysis of myoepithelial/basal cell markers in adenoid cystic carcinoma and polymorphous low-grade adenocarcinoma. Mod Pathol 2008;21:105-114.

25 Yemelyanova AV, Vang R, Judson K, et al. Distinction of primary and metastatic mucinous tumors involving the ovary: analysis of size and laterality data by primary site with reevaluation of an algorithm for tumor classification. Am J Surg Pathol 2008;32: 128-138.

26 Antila R, Jalkanen J, Heikinheimo O. Comparison of secondary and primary ovarian malignancies reveals differences in their pre- and perioperative characteristics. Gynecol Oncol 2006;101:97-101.

Supplementary Information accompanies the paper on Modern Pathology website (http://www.nature.com/ modpathol) 